\title{
Pinning Lur'e Complex Networks via Output Feedback Control
}

\author{
Fang Liu, ${ }^{1}$ Qiang Song, ${ }^{1}$ Jinde Cao, ${ }^{2,3}$ and Jianquan $\mathrm{Lu}^{2}$ \\ ${ }^{1}$ School of Information Engineering, Huanghuai University, Henan 463000, China \\ ${ }^{2}$ Department of Mathematics, Southeast University, Nanjing 210096, China \\ ${ }^{3}$ Department of Mathematics, Faculty of Science, King Abdulaziz University, Jeddah 21589, Saudi Arabia
}

Correspondence should be addressed to Qiang Song; qsongseu@gmail.com

Received 6 February 2014; Accepted 5 March 2014; Published 13 April 2014

Academic Editor: Guanghui Wen

Copyright (C) 2014 Fang Liu et al. This is an open access article distributed under the Creative Commons Attribution License, which permits unrestricted use, distribution, and reproduction in any medium, provided the original work is properly cited.

\begin{abstract}
Without requiring the full-state information of network nodes, this paper studies the pinning synchronization in a network of Lur'e dynamical systems based on the output feedback control strategy. Some simple pinning conditions are established for both undirected and directed Lur'e networks by using $M$-matrix theory and $S$-procedure technique. With the derived stability criteria, the pinning synchronization problem of large-scale Lur'e networks can be transformed to the test of a low-dimensional linear matrix inequality. Some remarks are further given to address the selection of pinned nodes and the design of pinning feedback gains. Numerical results are provided to demonstrate the effectiveness of the theoretical analysis.
\end{abstract}

\section{Introduction}

Lur'e systems refer to class of nonlinear dynamical systems which are formed by a linear system and a nonlinear feedback loop satisfying a sector condition. Actually, many nonlinear systems, such as Chua's circuit system and some hyperchaotic systems, can be described by Lur'e systems. Over the past few decades, much effort has been devoted to the research on Lur'e systems. The stability problem of Lur'e systems has been intensively studied, yielding some fundamental results such as the circle and Popov criteria [1-6]. The synchronization problem of Lur'e systems has also been deeply investigated for the purpose of secure communication and engineering applications [7-12].

Recently, the synchronization phenomena in complex dynamical networks with each node being a Lur'e system have attracted increasing attention. Liu et al. [13] addressed the global synchronization in coupled Lur'e systems based on the absolute stability theory. Ding and Han [14] studied the effect of the communication delay on the synchronization in Lur'e networks. Ji et al. [15] considered the synchronization problem for complex networks composed of time-delayed Lur'e systems. It is worth noting that the Lur'e networks in [13-15] achieved synchronization by local interactions among network nodes without involving any external force. When a
Lur'e network cannot reach synchronization by itself, some appropriate controllers may be designed such that the entire network can be synchronized to some desired trajectory.

For a complex network with a large number of nodes, it is literally impossible to apply control actions to all nodes due to the high control cost. Current studies have shown that the pinning control strategy [16-22] can be utilized to synchronize a network to a homogenous state, where local feedback injections are only placed on a small fraction of network nodes. The pinning control problem for Lur'e networks with undirected topologies has been investigated by some researchers [23-25]. More recently, Song et al. [26] developed some simple pinning conditions for Lur'e complex networks with directed topologies by using $M$-matrix $[27,28]$ and algebraic graph theories.

In the pinning control of complex networks, how to choose a set of pinned nodes is one of the most difficult problems. For an undirected and connected network, it is well-known that the network can be synchronized if a subset of nodes is either specifically or randomly pinned [16, 17]. As for a directed network, it has been proved that pinning control should be applied to the roots of a minimum number of directed spanning forests of the network topology [18, 19, 21]. Song and Cao [22] showed that the nodes whose out-degrees are bigger than their in-degrees must be chosen 
as pinned candidates. More recently, by using $M$-matrix theory, Song et al. [26, 29, 30] and Wen et al. [31] derived some stability criteria for pinning networked systems with directed topologies. In particular, some M-matrix strategies were developed to discuss several challenging problems in the pinning control of networked systems [30].

In most literature, the synchronization of complex networks is usually reached by using the full states of network nodes which may not always be available in many practical cases [32-35]. Note that some observer-based algorithms have been developed for achieving synchronization in complex networks $[36,37]$. In this paper, we investigate the pinning synchronization of Lur'e complex networks by utilizing the observed states, that is, output states, of network nodes. Without requiring full state information of network nodes, we propose a distributed output feedback control approach to pin Lur'e networks with node dynamics satisfying sector conditions. Then, by using $M$-matrix and algebraic graph theories, we derive some simple stability criteria to convert the pinning control problem of Lur'e networks into the test of a linear matrix inequality whose dimension is just determined by a single Lur'e node. Moreover, we discuss the selection of pinned nodes and the design of pinning feedback gains for both undirected and directed Lur'e complex networks.

The rest of this paper is organized as follows. In Section 2, some preliminaries are provided. Section 3 formulates the pinning control problem of Lur'e networks with output feedback coupling. Sections 4 and 5 derive some pinning conditions for undirected and directed Lur'e networks, respectively. In Section 6, numerical results are given to validate the theoretical analysis. Finally, some concluding remarks are stated in Section 7.

\section{Preliminaries}

In this section, we provide some mathematical preliminaries and some supporting lemmas to derive the main results of this paper.

2.1. Notations. The standard notations are used throughout this paper. Let $\mathbb{R}$ and $\mathbb{C}$ denote the sets of real and complex numbers, respectively. For $z \in \mathbb{C}, \operatorname{Re}(z)$ represents its real part. Let $I_{n}$ be the $n$-dimensional identity matrix and let $1_{n} \in$ $\mathbb{R}^{n}\left(0_{n} \in \mathbb{R}^{n}\right)$ be the vector of all ones (zeros). For a matrix $A \in \mathbb{R}^{n \times n}$, let $A^{T}$ be its transpose, $A^{-1}$ its inverse, $A_{s}=$ $\left(A+A^{T}\right) / 2$ its symmetric part, and $\lambda_{i}(A)$ the $i$ th eigenvalue, and let $\mathscr{J}(A)=\min _{1 \leq i \leq n} \operatorname{Re}\left(\lambda_{i}(A)\right)$ denote the minimum real part of all its eigenvalues. For a real symmetric matrix $X \in \mathbb{R}^{n \times n}$, let $\lambda_{\text {min }}(X)$ and $\lambda_{\text {max }}(X)$ be its minimum and maximum eigenvalues, respectively, and write $X>0(X<0)$ if $X$ is positive (negative) definite. The symbol $\otimes$ denotes the Kronecker product [27].

2.2. Graph Theory. The information interaction in a networked system can be described by a weighted graph $\mathscr{G}=$ $\{\mathscr{V}, \mathscr{E}, \mathscr{A}\}$ which is composed of a node set $\mathscr{V}=\{1, \ldots, N\}$, an edge set $\mathscr{E} \subseteq \mathscr{V} \times \mathscr{V}$, and an adjacency matrix $\mathscr{A}$. A directed edge between two different nodes is denoted by $(j, i)$ indicating that node $i$ can access the information from node $j$. The $(i, j)$ th entry of the adjacency matrix $\mathscr{A}$ is defined by $a_{i j}>0 \Leftrightarrow(j, i) \in \mathscr{E}(i \neq j)[29-31,38,39]$. In this paper, it is always assumed that $a_{i i}=0$ for all $i \in \mathscr{V}$. The elements of the Laplacian matrix $L=\left(l_{i j}\right)_{N \times N}$ associated with the adjacency matrix $\mathscr{A}$ are defined as follows:

$$
l_{i j}=-a_{i j} \leq 0 \quad \text { for } i \neq j, \quad l_{i i}=\sum_{k=1, k \neq i}^{N} a_{i k} .
$$

A directed path is a sequence of directed edges with distinct nodes. A digraph with $N$ nodes is called a directed tree if it has $N-1$ edges and there exists a node with directed paths to every other node. A digraph is called strongly connected if for any two different nodes $j$ and $i$, one can always find a directed path from node $j$ to node $i$. A digraph is said to have or contain a directed spanning tree if there exists at least one node having a directed path to every other node [29-31, 38, 39].

2.3. M-Matrix Theory. Some results related to $M$-matrix will be used to study the pinning synchronization of Lur'e complex networks.

Definition 1 (see $[27,28])$. A nonsingular matrix $A=\left(a_{i j}\right) \epsilon$ $\mathbb{R}^{n \times n}$ is called an $M$-matrix if $a_{i j} \leq 0$ whenever $i \neq j$ and all elements of $A^{-1}$ are nonnegative.

Lemma 2 (see $[27,28]$ ). For a nonsingular matrix $A=$ $\left(a_{i j}\right)_{n \times n} \in \mathbb{R}^{n \times n}$ with $a_{i j} \leq 0(i \neq j)$, the following statements are equivalent:

(1) $A$ is an M-matrix;

(2) all eigenvalues of $A$ have positive real parts; that is, $\operatorname{Re}\left(\lambda_{i}(A)\right)>0$ for all $i=1, \ldots, n$;

(3) there exists a positive definite diagonal matrix $\Xi=$ $\operatorname{diag}\left(\xi_{1}, \ldots, \xi_{n}\right)>0$ such that $\Xi A+A^{T} \Xi$ is positive definite.

2.4. Some Supporting Lemmas. Some properties of the Kronecker product are listed as follows.

Lemma 3 (see [27]). For matrices $A, B, C$, and $D$ with appropriate dimensions, one has

(1) $(A \otimes B)^{T}=A^{T} \otimes B^{T}$;

(2) $(A+B) \otimes C=A \otimes C+B \otimes C$;

(3) $(A \otimes B)(C \otimes D)=(A C) \otimes(B D)$.

Lemma 4 (see [40]). Let $A \in \mathbb{R}^{n \times n}$ be symmetric. One has $\lambda_{\min }(A) x^{T} x \leq x^{T} A x \leq \lambda_{\max }(A) x^{T} x$, forall $x \in R^{n}$.

The $S$-procedure technique [1] which is widely used to study the stability problem of Lur'e dynamical systems can be stated as follows.

Lemma 5 (see [1]). Let $V_{0}(x), V_{1}(x), \ldots, V_{m}(x)$ be quadratic forms over $x \in \mathbb{R}^{n}$. One has $V_{0}(x)<0$ for all $x \neq 0_{n}$ satisfying 
$V_{i}(x) \leq 0, i=1 \ldots, m$, if there exist some nonnegative numbers $\tau_{1}, \ldots, \tau_{m} \geq 0$ such that $V_{0}(x)-\sum_{i=1}^{m} \tau_{i} V_{i}(x)<0$.

Lemma 6 (Schur complement [1]). The following linear matrix inequality (LMI)

$$
\left(\begin{array}{ll}
Q(x) & S(x) \\
S(x)^{T} & R(x)
\end{array}\right)>0
$$

where $Q(x)=Q(x)^{T}, R(x)=R(x)^{T}$ is equivalent to either of the following conditions:

(1) $Q(x)>0, R(x)-S(x)^{T} Q(x)^{-1} S(x)>0$;

(2) $R(x)>0, Q(x)-S(x) R(x)^{-1} S(x)^{T}>0$.

\section{Output Feedback Control Algorithm for Pinning Lur'e Networks}

Consider a complex network with each node being a Lur'e dynamical system described by

$$
\begin{gathered}
\dot{x}_{i}(t)=A x_{i}+B f\left(C x_{i}\right), \\
y_{i}(t)=C x_{i}(t), \quad i=1, \ldots, N,
\end{gathered}
$$

where $x_{i}=\left(x_{i 1}, \ldots, x_{i n}\right)^{T}$ is the state variable of the $i$ th node, $y_{i}=\left(y_{i 1}, \ldots, y_{i m}\right)^{T}$ is the output state of the $i$ th node, and $A \in$ $\mathbb{R}^{n \times n}, B \in \mathbb{R}^{n \times m}, C \in \mathbb{R}^{m \times n}$ with $C_{k}^{T}$ being its $k$ th row; the nonlinear vector-valued function $f\left(C x_{i}\right)=\left(f_{1}\left(C_{1}^{T} x_{i}\right), \ldots\right.$, $\left.f_{m}\left(C_{m}^{T} x_{i}\right)\right)^{T} \in \mathbb{R}^{m}$ satisfies the following sector conditions:

$$
0 \leq \frac{f_{k}(b)-f_{k}(a)}{b-a} \leq \delta_{k}, \quad \forall a, b \in \mathbb{R}, a \neq b, k=1, \ldots, m .
$$

In many practical cases, the full states of network nodes are not always available. By utilizing the output states of network nodes, we consider the following Lur'e network model:

$$
\begin{gathered}
\dot{x}_{i}(t)=A x_{i}+B f\left(C x_{i}\right)-\sigma \sum_{j=1}^{N} l_{i j} F y_{j}+u_{i}, \\
y_{i}(t)=C x_{i}(t), \quad i=1, \ldots, N
\end{gathered}
$$

where $\sigma>0$ is the coupling strength, $l_{i j}$ is the $(i, j)$ th entry of Laplacian matrix $L \in \mathbb{R}^{N \times N}, F \in \mathbb{R}^{n \times m}$ is the output feedback gain matrix, and $u_{i}$ is the control input to be designed.

Note that the isolated node (or leader node) for Lur'e complex network (5) is given by

$$
\begin{gathered}
\dot{x}_{r}(t)=A x_{r}(t)+B f\left(C x_{r}\right), \\
y_{r}(t)=C x_{r}(t),
\end{gathered}
$$

where $x_{r}=\left(x_{r 1}, \ldots, x_{r n}\right)^{T}$ and $y_{r}=\left(y_{r 1}, \ldots, y_{r m}\right)^{T}$.

Definition 7. The Lur'e complex network (5) is said to be globally asymptotically synchronized to the isolated node (6); that is, $x_{i}(t) \rightarrow x_{r}(t), i=1, \ldots, N$, as $t \rightarrow \infty$, for any initial condition.

To reduce the number of controllers, one can adopt the pinning control strategy to synchronize complex network (5) to the isolated node (6). For Lur'e network (5), let $\mathscr{V}=$ $\{1, \ldots, N\}$ and $\mathscr{V}_{\text {pin }}=\left\{i_{1}, \ldots, i_{l}\right\} \subset \mathscr{V}$ be the sets of total and pinned nodes, respectively, where $1 \leq l<N$. Applying pinning control to network (5) yields

$$
\begin{gathered}
\dot{x}_{i}(t)=A x_{i}+B f\left(C x_{i}\right)-\sigma \sum_{j=1}^{N} l_{i j} F y_{j}-\sigma d_{i} F\left(y_{i}-y_{r}\right), \\
y_{i}(t)=C x_{i}(t), \quad i=1, \ldots, N
\end{gathered}
$$

where the pinning feedback gains are defined as follows:

$$
d_{i}>0 \quad \text { if } i \in \mathscr{V}_{\text {pin }}, \quad d_{i}=0 \quad \text { if } i \in \mathscr{V} \backslash \mathscr{V}_{\text {pin }} .
$$

Suppose that the matrix pair $(A, C)$ in complex network (7) is detectable, and the output feedback gain matrix is designed as $F=P^{-1} C^{T}$, where $P \in \mathbb{R}^{n \times n}$ is a positive definite matrix to be determined later. Then the pinning-controlled Lur'e network (7) becomes

$$
\begin{aligned}
& \dot{x}_{i}(t)= A x_{i}(t)+B f\left(C x_{i}\right)-\sigma \sum_{j=1}^{N} l_{i j} P^{-1} C^{T} C x_{j} \\
&-\sigma d_{i} P^{-1} C^{T} C\left(x_{i}-x_{r}\right), \\
& y_{i}(t)=C x_{i}(t), \quad i=1, \ldots, N .
\end{aligned}
$$

Remark 8. If the matrix pair $(A, C)$ is detectable, there always exists a matrix $F \in \mathbb{R}^{n \times m}$ to ensure that $A-F C$ is a Hurwitz matrix. Moreover, one can always find a positive definite matrix $P \in \mathbb{R}^{n \times n}$ and a positive scalar $\tau>0$ such that $P A+A^{T} P-\tau C^{T} C<0[1]$.

Remark 9. In most literature, the pinning control approaches for complex networks usually require full states of network nodes $[18-22,29,30]$. Note that the pinning control algorithm (9) is actually implemented by utilizing the output states of network nodes.

For the convenience of our discussions in this paper, let $L$ be the Laplacian matrix of network (5), $D=\operatorname{diag}\left(d_{1}, \ldots, d_{N}\right)$ the matrix of pinning feedback gains defined by (8), and $\Delta=$ $\operatorname{diag}\left(\delta_{1}, \ldots, \delta_{m}\right)$ the matrix describing the sector condition (4).

Let $e_{i}(t)=x_{i}(t)-x_{r}(t)$ and $\eta_{i}=\left(\eta_{i 1}, \ldots, \eta_{i m}\right)^{T}=f\left(C x_{i}\right)-$ $f\left(C x_{r}\right), i=1, \ldots, N$. Then by $(4)$, we have $\eta_{i k}=f_{k}\left(C_{k}^{T} x_{i}\right)-$ $f_{k}\left(C_{k}^{T} x_{r}\right)$ satisfying

$$
0 \leq \frac{\eta_{i k}}{C_{k}^{T} e_{i}} \leq \delta_{k}, \quad k=1, \ldots, m,
$$

which is equivalent to

$$
\eta_{i k}\left(\eta_{i k}-\delta_{k} C_{k}^{T} e_{i}\right) \leq 0, \quad k=1, \ldots, m .
$$


From (6) and (9), we obtain the following error system:

$$
\begin{array}{r}
\dot{e}_{i}(t)=A e_{i}+B \eta_{i}-\sigma \sum_{j=1}^{N} l_{i j} P^{-1} C^{T} C e_{j}-\sigma d_{i} P^{-1} C^{T} C e_{i} \\
i=1, \ldots, N .
\end{array}
$$

Let $e(t)=\left(e_{1}^{T}(t), \ldots, e_{N}^{T}(t)\right)^{T}$ and $\eta=\left(\eta_{1}^{T}, \ldots, \eta_{N}^{T}\right)^{T}$. Rewrite (12) in the matrix form as

$$
\dot{e}(t)=\left(I_{N} \otimes A\right) e+\left(I_{N} \otimes B\right) \eta-\left(\sigma(L+D) \otimes\left(P^{-1} C^{T} C\right)\right) e .
$$

By using $M$-matrix theory, Song et al. $[29,30]$ have shown that $\mathscr{J}(L+D)$, that is, $\min _{1 \leq i \leq N} \operatorname{Re}\left(\lambda_{i}(L+D)\right)$ plays an important role in investigating the pinning control of networked systems. The following result is useful to study the synchronization problem of Lur'e network (9).

Lemma 10 (see [30]). Let $\widetilde{\mathscr{G}}$ be the augmented digraph formed by the nodes of Lur'e network (5) and the isolated node (6). One has $\mathscr{J}(L+D)>0$ if and only if $\widetilde{\mathscr{G}}$ contains a directed spanning tree, which means that the isolated node (6) has a directed path to every other network node.

Remark 11. By Lemmas 2 and 10 and the definition of $L$ in (1), one knows that $L+D$ is an $M$-matrix if and only if $\widetilde{\mathscr{G}}$ contains a directed spanning tree. Moreover, $L+D$ is positive definite if $L$ is symmetric.

\section{Pinning Criteria for Undirected Lur'e Network}

In this section, we consider the pinning synchronization of Lur'e network (9) with undirected topology and derive some simple pinning conditions for the network by using linear matrix inequality (LMI) and the $S$-procedure $[1,5,8]$.

Theorem 12. Suppose that $L=L^{T}$ and there exist a positive definite matrix $P \in \mathbb{R}^{n \times n}$ and a matrix $T=\operatorname{diag}\left(\tau_{1}, \ldots, \tau_{m}\right)>$ 0 such that

$$
Q=\left(\begin{array}{cc}
P A+A^{T} P-2 \sigma \lambda_{1} C^{T} C & P B+C^{T} \Delta T \\
B^{T} P+T \Delta C & -2 T
\end{array}\right)<0
$$

where $\lambda_{1}=\lambda_{\min }(L+D)>0$. Then, the pinning-controlled Lur'e network (9) globally asymptotically synchronizes to the isolated node (6).

Proof. Since $L+D$ is a real symmetric matrix, one knows that all eigenvalues of $L+D$ are real. It follows from the condition of the theorem that the minimum eigenvalue of $L+D$ is a positive number; that is, $\lambda_{1}=\lambda_{\text {min }}(L+D)>0$. Then we see that $L+D$ is a positive definite matrix. By Lemma 4 , we have $L+D \geq \lambda_{1} I_{N}>0$.
From sector condition (11) and $\tau_{k}>0, k=1, \ldots, m$, we obtain

$$
\sum_{k=1}^{m} \tau_{k} \eta_{i k}\left(\eta_{i k}-\delta_{k} C_{k}^{T} e_{i}\right) \leq 0,
$$

which is equivalent to

$$
\eta_{i}^{T}\left(T \eta_{i}-T \Delta C e_{i}\right) \leq 0, \quad \forall i \in\{1, \ldots, N\} .
$$

Take the Lyapunov function candidate as

$$
V(t)=\frac{1}{2} e^{T}(t)\left(I_{N} \otimes P\right) e(t),
$$

where $P>0$ satisfies condition (14).

Considering Lemma 3, inequality (16), and the $S$ procedure stated in Lemma 5, we calculate the time derivative of $V(t)$ along the trajectory of error system (13) as follows:

$$
\begin{aligned}
\dot{V}(t)= & \sum_{i=1}^{N} e_{i}^{T} P A e_{i}+\sum_{i=1}^{N} e_{i}^{T} P B \eta_{i}-e^{T}\left[(\sigma(L+D)) \otimes\left(C^{T} C\right)\right] e \\
\leq & \sum_{i=1}^{N} e_{i}^{T} P A e_{i}+\sum_{i=1}^{N} e_{i}^{T} P B \eta_{i} \\
& -e^{T}\left[(\sigma(L+D)) \otimes\left(C^{T} C\right)\right] e-\sum_{i=1}^{N} \eta_{i}^{T}\left(T \eta_{i}-T \Delta C e_{i}\right) \\
\leq & \sum_{i=1}^{N}\left(e_{i}^{T} P A e_{i}+e_{i}^{T} P B \eta_{i}\right)-e^{T}\left[\left(\sigma \lambda_{1} I_{N}\right) \otimes\left(C^{T} C\right)\right] e \\
& -\sum_{i=1}^{N} \eta_{i}^{T}\left(T \eta_{i}-T \Delta C e_{i}\right) \\
= & \sum_{i=1}^{N}\left(e_{i}^{T} P A e_{i}-\sigma \lambda_{1} e_{i}^{T} C^{T} C e_{i}+e_{i}^{T} P B \eta_{i}\right. \\
& \left.+e_{i}^{T} C^{T} \Delta T \eta_{i}-\eta_{i}^{T} T \eta_{i}\right) \\
= & \frac{1}{2} \sum_{i=1}^{N} z_{i}^{T} Q z_{i}=\frac{1}{2} z^{T}(t)\left(I_{N} \otimes Q\right) z(t)
\end{aligned}
$$

where $z_{i}=\left(e_{i}^{T}, \eta_{i}^{T}\right)^{T}, z(t)=\left(z_{1}^{T}(t), \ldots, z_{N}^{T}(t)\right)^{T}$, and $Q$ is defined in (14).

By (14) and (18), we know that $\dot{V}(t) \leq 0$ and $\dot{V}(t) \equiv 0$ if and only if $z(t)=0_{N(n+m)}$. Thus, the set $\mathscr{M}=\{z(t)$ | $\left.z(t)=0_{N(n+m)}\right\}$ is the largest invariant set contained in the set $\mathscr{D}=\{z(t) \mid \dot{V}(t) \equiv 0\}$ for error system (13). According to LaSalle's invariance principle [5], starting from any initial condition, every solution of error system (13) approaches $\mathscr{M}$ as $t \rightarrow \infty$, which indicates $e_{i}(t) \rightarrow 0, i=1, \ldots, N$. Then the error system (13) is asymptotically stable at the origin. Therefore, the pinning-controlled Lur'e network (9) globally asymptotically synchronizes to the isolated node (6).

Remark 13. Note that the dimension of LMI condition (14) is equal to $n+m$ determined by the single Lur'e node. 
Remark 14. In Remark 8, we have pointed out that one can find a positive definite matrix $P \in \mathbb{R}^{n \times n}$ and a scalar $\tau>0$ such that $P A+A^{T} P-\tau C^{T} C<0$ if the pair $(A, C)$ is detectable. In view of Lemma 6 , we see that the detectability of $(A, C)$ provides a necessary condition for the solvability of LMI (14).

Remark 15. From condition (14) in Theorem 12, we know that the quantity $\lambda_{\min }(L+D)$ is very important to study the synchronization in undirected Lur'e network (9). Suppose that the network topology is composed of several disconnected components. By Lemma 10 and Remark 11, at least one node in each component should be pinned such that $L+D$ is positive definite, and the pinned nodes can be randomly or specifically chosen. For better pinning control performance, one can pin the most highly connected nodes to achieve larger $\lambda_{\text {min }}(L+D)$ such that condition (14) can be satisfied more easily.

\section{Pinning Criteria for Directed Lur'e Network}

In this section, we study the pinning synchronization of Lur'e network (9) with directed topology. Due to asymmetric coupling, the pinning synchronization problem of directed complex networks is more difficult than that of undirected networks. M-matrix theory will be adopted to derive some pinning conditions for directed Lur'e network (9).

Theorem 16. Suppose that $L$ is not symmetric. Assume that a positive definite matrix $P \in \mathbb{R}^{n \times n}$ and a matrix $T=$ $\operatorname{diag}\left(\tau_{1}, \ldots, \tau_{m}\right)>0$ can be found such that

$$
R=\left(\begin{array}{cc}
P A+A^{T} P-2 \rho C^{T} C & P B+C^{T} \Delta T \\
B^{T} P+T \Delta C & -2 T
\end{array}\right)<0,
$$

where $\rho$ is a positive scalar subject to

$$
0<\rho<\sigma \mathscr{J}(L+D)
$$

Then, Lur'e network (9) globally asymptotically synchronizes to the isolated node (6).

Proof. Let $\lambda_{i}$ be the $i$ th eigenvalue of $L+D$. It is easy to verify that $\sigma \lambda_{i}-\rho$ is the $i$ th eigenvalue of $\sigma(L+D)-\rho I_{N}$. It follows from condition (20) that $\operatorname{Re}\left(\sigma \lambda_{i}-\rho\right)>0$ holds for all $i=$ $1, \ldots, N$. Then, by the definition of $L$ in (1) and Lemma 2, we know that $\sigma(L+D)-\rho I_{N}$ is an $M$-matrix and there exists a positive definite diagonal matrix $\Xi=\operatorname{diag}\left(\xi_{1}, \ldots, \xi_{N}\right)>0$ such that

$$
\left[\Xi\left(\sigma(L+D)-\rho I_{N}\right)\right]_{s}>0 .
$$

Construct the following Lyapunov function candidate:

$$
V(t)=\frac{1}{2} e^{T}(t)(\Xi \otimes P) e(t),
$$

where $P$ satisfies condition (19).
The time derivative of $V(t)$ along the trajectory of error system (13) yields

$$
\begin{aligned}
\dot{V}(t)= & \sum_{i=1}^{N} \xi_{i} e_{i}^{T} P A e_{i}+\sum_{i=1}^{N} \xi_{i} e_{i}^{T} P B \eta_{i} \\
& -e^{T}\left[(\sigma \Xi(L+D)) \otimes\left(C^{T} C\right)\right] e \\
\leq & \sum_{i=1}^{N} \xi_{i} e_{i}^{T} P A e_{i}+\sum_{i=1}^{N} \xi_{i} e_{i}^{T} P B \eta_{i} \\
& -e^{T}\left[(\sigma \Xi(L+D)) \otimes\left(C^{T} C\right)\right] e \\
& -\sum_{i=1}^{N} \xi_{i} \eta_{i}^{T}\left(T \eta_{i}-T \Delta C e_{i}\right) \\
= & \sum_{i=1}^{N} \xi_{i}\left(e_{i}^{T} P A e_{i}+e_{i}^{T} P B \eta_{i}+e_{i}^{T} C^{T} \Delta T \eta_{i}-\eta_{i}^{T} T \eta_{i}\right) \\
& -\rho \sum_{i=1}^{N} \xi_{i} e_{i}^{T} C^{T} C e_{i}+\rho \sum_{i=1}^{N} \xi_{i} e_{i}^{T} C^{T} C e_{i} \\
& -e^{T}\left[(\sigma \Xi(L+D)) \otimes\left(C^{T} C\right)\right] e \\
\leq & \frac{1}{2} z^{T}(t)(\Xi \otimes R) e(t), \\
& 1 \sum_{i=1}^{N} \xi_{i} z_{i}^{T} R z_{i}\left(\left[\Xi\left(\sigma(L+D)-\rho I_{N}\right)\right]_{s} \otimes\left(C^{T} C\right)\right) e \\
& \\
& \\
&
\end{aligned}
$$

where the first inequality is obtained by applying inequality (16), the last inequality follows (21) and the fact $C^{T} C \geq 0$, $z_{i}=\left(e_{i}^{T}, \eta_{i}^{T}\right)^{T}, z(t)=\left(z_{1}^{T}(t), \ldots, z_{N}^{T}(t)\right)^{T}$, and $R$ is defined in (19).

By LaSalle's invariance principle, we can show that $e_{i}(t) \rightarrow 0, i=1, \ldots, N$, which indicates that the pinning-controlled Lur'e network (9) globally asymptotically synchronizes to the isolated node (6).

Remark 17. From condition (20) in Theorem 16, we know that a set of pinned nodes should be selected to ensure $\mathscr{J}(L+$ D) $>0$. For a directed network, suppose that the multiplicity of the zero eigenvalue of $L$ is equal to $p$. Song et al. [30] decomposed the network topology into $p$ components, where each component has a directed tree. By Lemma 10, at least one root node in each component should be pinned such that $L+$ $D$ is an $M$-matrix, which means that the minimum number of pinned nodes is $p$. It is worth mentioning that the remaining $N-p$ nodes can be rearranged in descending order according to the differences of their out-degrees and in-degrees [29]. When the network topology contains a directed spanning tree, the Laplacian matrix $L$ has a simple zero eigenvalue [38]; that is, $p=1$. Then it is possible to pin Lur'e network (9) if the coupling strength $\sigma$ is sufficiently large in view of conditions (19) and (20), which also confirms the pioneering work in [18]. 


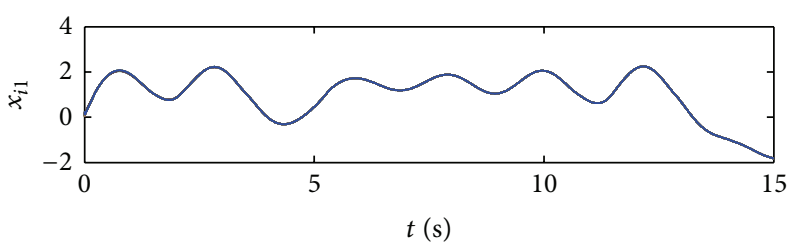

(a)

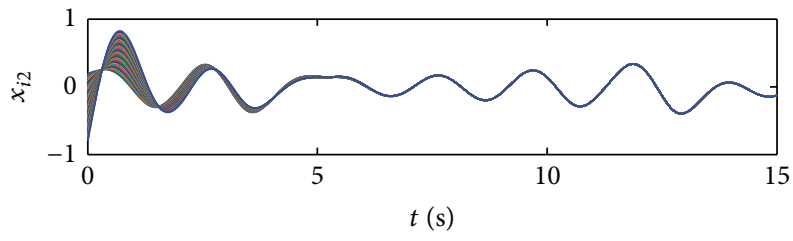

(b)

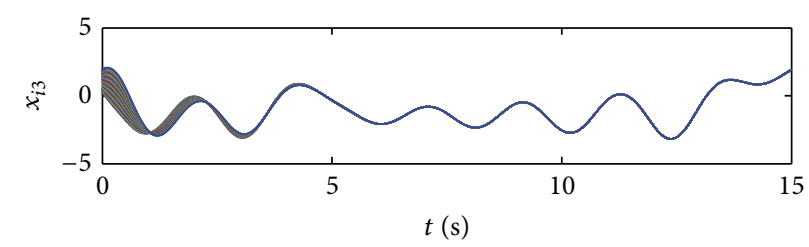

(c)

FIGURE 1: State evolutions of pinning-controlled Lur'e network.

Remark 18. Song et al. [30] have shown that $\mathscr{J}(L+D)$ monotonically increases with respect to the number of pinned nodes or pinning feedback gains. However, $\mathscr{J}(L+$ $D) \leq \mathscr{J}\left(L\left(\mathscr{V} \backslash \mathscr{V}_{\text {pin }}\right)\right)$ always holds even if the pinning feedback gains are sufficiently large. Therefore, it would be desirable to synchronize Lur'e network (9) by pinning a least number of nodes with relatively lower control gains.

Remark 19. One may use $\lambda_{\min }\left(\Xi(L+D)+(L+D)^{T} \Xi\right)$ to replace the parameter $\rho$ in (19) to derive pinning condition for directed Lur'e networks, which will involve the computation of the matrix $\Xi$. Moreover, it will be quite difficult to discuss the selection of pinned nodes and the design of pinning feedback gains. Thus, we intentionally introduce the parameter $\rho$ in Theorem 16 as a transitional variable to derive condition (19). Treating $\rho$ as a scalar matrix, we can solve LMIs (19) and (20) with Matlab LMI toolbox to obtain appropriate parameters $\rho$ and $P$. If no feasible solution is found, we can gradually increase the pinning feedback gains or continually add more nodes to $\mathscr{V}_{\text {pin }}$ to increase $\mathscr{J}(L+D)$ until conditions (19) and (20) are satisfied.

\section{Numerical Results}

In this section, some simulation results are given to verify our theoretical analysis. For a complex network with $N$ nodes, the quantity $E(t)=\sqrt{(1 / N) \sum_{i=1}^{N} e_{i}^{T}(t) e_{i}(t)}$ is used to measure the quality of the pinning process $[18,22]$.

Consider a Lur'e complex network in the form of (5) composed of fifty nodes:

$$
\begin{gathered}
\dot{x}_{i}(t)=A x_{i}+B f\left(C x_{i}\right)-\sigma \sum_{j=1}^{50} l_{i j} F y_{j}+u_{i}, \\
y_{i}(t)=C x_{i}(t), \quad i=1, \ldots, 50
\end{gathered}
$$

where $x_{i}=\left(x_{i 1}, x_{i 2}, x_{i 3}\right)^{T}$ is the state variable of the $i$ th node, $\sigma=5$,

$$
\begin{gathered}
A=\left(\begin{array}{ccc}
-a m_{1} & a & 0 \\
1 & -1 & 1 \\
0 & -b & 0
\end{array}\right), \quad B=\left(\begin{array}{c}
-a\left(m_{0}-m_{1}\right) \\
0 \\
0
\end{array}\right), \\
C=\left(\begin{array}{lll}
1 & 0 & 0
\end{array}\right),
\end{gathered}
$$

with $a=9, b=14.28, m_{0}=-1 / 7$ and $m_{1}=2 / 7$; the nonlinear function is given by

$$
f\left(C x_{i}\right)=\frac{1}{2}\left(\left|x_{i 1}+1\right|-\left|x_{i 1}-1\right|\right) .
$$

The interaction digraph $\mathscr{G}$ of Lur'e network (24) is determined by a directed scale-free network generated by using the techniques in [20]. According to [20], $\mathscr{G}$ is strongly connected and thus contains a directed spanning tree. Based on Remark 17, we know that the minimum number of pinned nodes is one and any node can be chosen to be pinned. When node 2 is pinned with control gain $d_{2}=1.0$, we have

$$
\begin{gathered}
\mathscr{J}(L+D)=0.0912, \\
\sigma \mathscr{J}(L+D)=5 \times 0.0912=0.4560 .
\end{gathered}
$$

It is easy to verify that the matrix pair $(A, C)$ is detectable, and the nonlinear function $f$ belongs to the sector $[0,1]$ which means that $\Delta=1$. Solving LMIs (19) and (20) in Theorem 16, we obtain the following feasible solution:

$$
\begin{gathered}
\rho=0.3470, \quad T=0.8556 \\
P=\left(\begin{array}{ccc}
0.1141 & -0.1179 & 0.0944 \\
-0.1179 & 0.8047 & -0.0776 \\
0.0944 & -0.0776 & 0.1110
\end{array}\right) .
\end{gathered}
$$

With the output feedback control algorithm (9), we apply pinning control to node 2 of Lur'e network (24). The time evolutions of network state and synchronization error are shown in Figures 1 and 2, respectively. We can clearly see that the Lur'e network with fifty nodes is successfully synchronized to a homogenous state by pinning a single network node. 


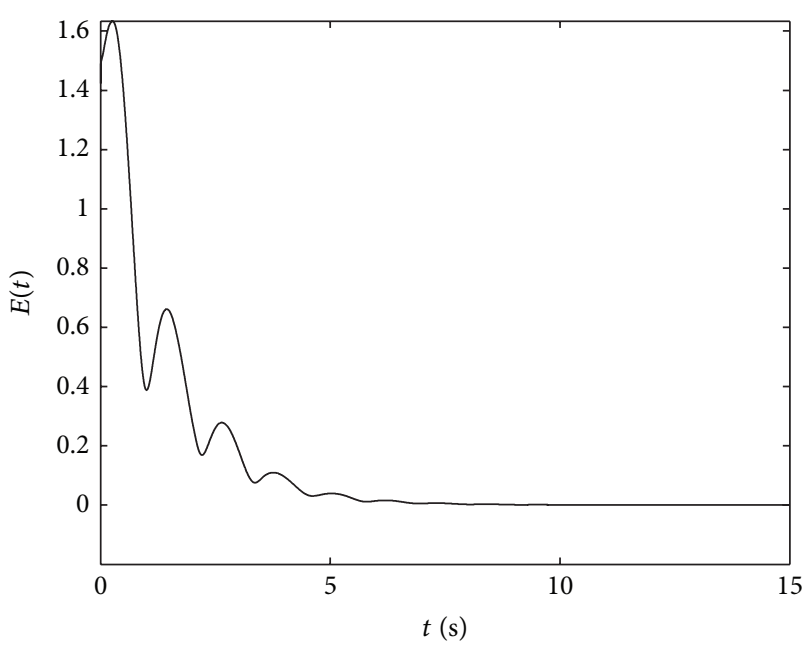

Figure 2: Pinning synchronization error of Lur'e network.

\section{Conclusions}

This paper proposes a distributed output feedback control approach to investigate the pinning synchronization in Lur'e complex networks. By using the tools from M-matrix theory, Lyapunov functional method, and observer techniques for nonlinear systems, some simple pinning criteria in terms of low-dimensional LMIs, whose dimensions are just determined by that of the single Lur'e node, have been derived for Lur'e networks with undirected and directed topologies, respectively. Numerical simulations have validated the effectiveness of the theoretical analysis. Since the time delay is inevitable in natural and physical systems, in the near future, it would be of interest to study the pinning control problem for Lur'e networks with both discrete and distributed timedelayed coupling on the basis of output feedback control strategies.

\section{Conflict of Interests}

The authors declare that there is no conflict of interests regarding the publication of this paper.

\section{Acknowledgments}

This work was jointly supported by the National Science Foundation of China under Grants no. 61273218, 61304172, 61272530 , and 61175119 and the Natural Science Foundation of Henan Province of China under Grant nos. 122102210027, 122300410220 , and 12B480005.

\section{References}

[1] S. Boyd, L. El Ghaoui, E. Feron, and V. Balakrishnan, Linear Matrix Inequalities in System and Control Theory, vol. 15, SIAM, Philadelphia, Pa, USA, 1994.

[2] W. M. Haddad and V. Kapila, "Absolute stability criteria for multiple slope-restricted monotonic nonlinearities," IEEE
Transactions on Automatic Control, vol. 40, no. 2, pp. 361-365, 1995.

[3] P. Park, "A revisited Popov criterion for nonlinear Lur'e systems with sector-restrictions," International Journal of Control, vol. 68, no. 3, pp. 461-469, 1997.

[4] J. A. K. Suykens, J. Vandewalle, and B. De Moor, "An absolute stability criterion for the Lur'e problem with sector and slope restricted nonlinearities," IEEE Transactions on Circuits and Systems I, vol. 45, no. 9, pp. 1007-1009, 1998.

[5] H. K. Khalil, Nonlinear Systems, Prentice Hall, Englewood Cliffs, NJ, USA, 3rd edition, 2002.

[6] C. A. C. Gonzaga, M. Jungers, and J. Daafouz, "Stability analysis of discrete-time Lur'e systems," Automatica, vol. 48, no. 9, pp. 2277-2283, 2012.

[7] J. A. K. Suykens, P. F. Curran, and L. O. Chua, "Robust synthesis for master-slave synchronization of Lur'e systems," IEEE Transactions on Circuits and Systems I: Fundamental Theory and Applications, vol. 46, no. 7, pp. 841-850, 1999.

[8] M. E. Yalçin, J. A. K. Suykens, and J. Vandewalle, "Master-slave synchronization of Lur'e systems with time-delay," International Journal of Bifurcation and Chaos in Applied Sciences and Engineering, vol. 11, no. 6, pp. 1707-1722, 2001.

[9] X. Huang, J. Cao, and D. W. C. Ho, "Synchronization criteria for Lur'e systems by dynamic output feedback with timedelay," International Journal of Bifurcation and Chaos in Applied Sciences and Engineering, vol. 16, no. 8, pp. 2293-2307, 2006.

[10] H. Huijberts, H. Nijmeijer, and T. Oguchi, "Anticipating synchronization of chaotic Lur'e systems," Chaos, vol. 17, no. 1, Article ID 013117, 2007.

[11] J. G. Lu and D. J. Hill, "Impulsive synchronization of chaotic Lur'e systems by linear static measurement feedback: An LMI approach," IEEE Transactions on Circuits and Systems II: Express Briefs, vol. 54, no. 8, pp. 710-714, 2007.

[12] J. Lu, J. Cao, and D. W. C. Ho, "Adaptive stabilization and synchronization for chaotic Lur'e systems with time-varying delay," IEEE Transactions on Circuits and Systems I: Regular Papers, vol. 55, no. 5, pp. 1347-1356, 2008.

[13] X. Liu, J. Wang, and L. Huang, "Global synchronization for a class of dynamical complex networks," Physica A: Statistical Mechanics and Its Applications, vol. 386, no. 1, pp. 543-556, 2007.

[14] K. Ding and Q.-L. Han, "Effects of coupling delays on synchronization in Lur'e complex dynamical networks," International Journal of Bifurcation and Chaos, vol. 20, no. 11, pp. 3565-3584, 2010.

[15] D. H. Ji, J. H. Park, W. J. Yoo, S. C. Won, and S. M. Lee, "Synchronization criterion for Lur'e type complex dynamical networks with time-varying delay," Physics Letters A: General, Atomic and Solid State Physics, vol. 374, no. 10, pp. 1218-1227, 2010.

[16] X. F. Wang and G. Chen, "Pinning control of scale-free dynamical networks," Physica A: Statistical Mechanics and Its Applications, vol. 310, no. 3-4, pp. 521-531, 2002.

[17] X. Li, X. Wang, and G. Chen, "Pinning a complex dynamical network to its equilibrium," IEEE Transactions on Circuits and Systems I: Regular Papers, vol. 51, no. 10, pp. 2074-2087, 2004.

[18] T. Chen, X. Liu, and W. Lu, "Pinning complex networks by a single controller," IEEE Transactions on Circuits and Systems I: Regular Papers, vol. 54, no. 6, pp. 1317-1326, 2007.

[19] C. W. Wu, "Localization of effective pinning control in complex networks of dynamical systems," in Proceedings of the IEEE International Symposium on Circuits and Systems (ISCAS '08), pp. 2530-2533, Seattle, Wash, USA, May 2008. 
[20] Y. Y. Lu and X. F. Wang, "Pinning control of directed dynamical networks based on ControlRank," International Journal of Computer Mathematics, vol. 85, no. 8, pp. 1279-1286, 2008.

[21] J. Lu, D. W. C. Ho, and Z. Wang, "Pinning stabilization of linearly coupled stochastic neural networks via minimum number of controllers," IEEE Transactions on Neural Networks, vol. 20, no. 10, pp. 1617-1629, 2009.

[22] Q. Song and J. Cao, "On pinning synchronization of directed and undirected complex dynamical networks," IEEE Transactions on Circuits and Systems I: Regular Papers, vol. 57, no. 3, pp. 672-680, 2010.

[23] Q. Z. Zhang and Z. K. Li, "Pinning control of complex Lur'e networks," Chinese Physics B, vol. 18, no. 6, pp. 2176-2183, 2009.

[24] Z. Li, Z. Duan, and G. Chen, "Global synchronised regions of linearly coupled Lur'e systems," International Journal of Control, vol. 84, no. 2, pp. 216-227, 2011.

[25] P. DeLellis and M. di Bernardo, "Adaptive pinning control of complex networks of Lur'e systems," in Proceedings of the 51st IEEE Conference on Decision and Control, pp. 6060-6064, Maui, Hawaii, USA, 2012.

[26] Q. Song, F. Liu, J. Cao, and J. Lu, "Some simple criteria for pinning a Lur'e network with directed topology," IET Control Theory and Applications, vol. 8, no. 2, pp. 131-138, 2014.

[27] R. A. Horn and C. R. Johnson, Topics in Matrix Analysis, Cambridge University Press, Cambridge, UK, 1991.

[28] A. Berman and R. J. Plemmons, Nonnegative Matrices in the Mathematical Sciences, vol. 9, SIAM, Philadelphia, Pa, USA, 1994.

[29] Q. Song, F. Liu, J. Cao, and W. Yu, "Pinning-controllability analysis of complex networks: an M-matrix approach," IEEE Transactions on Circuits and Systems I: Regular Papers, vol. 59, no. 11, pp. 2692-2701, 2012.

[30] Q. Song, F. Liu, J. Cao, and W. Yu, "M-matrix strategies for pinning-controlled leader-following consensus in multiagent systems with nonlinear dynamics," IEEE Transactions on Cybernetics, vol. 43, no. 6, pp. 1688-1697, 2013.

[31] G. Wen, Z. Duan, G. Chen, and W. Yu, "Consensus tracking of multi-agent systems with Lipschitz-type node dynamics and switching topologies," IEEE Transactions on Circuits and Systems I, vol. 61, no. 2, pp. 499-511, 2014.

[32] W. Lu and T. Chen, "New approach to synchronization analysis of linearly coupled ordinary differential systems," Physica D: Nonlinear Phenomena, vol. 213, no. 2, pp. 214-230, 2006.

[33] A. Arenas, A. Díaz-Guilera, J. Kurths, Y. Moreno, and C. Zhou, "Synchronization in complex networks," Physics Reports, vol. 469, no. 3, pp. 93-153, 2008.

[34] J. Lu, D. W. C. Ho, and J. Cao, "Synchronization in an array of nonlinearly coupled chaotic neural networks with delay coupling," International Journal of Bifurcation and Chaos in Applied Sciences and Engineering, vol. 18, no. 10, pp. 3101-3111, 2008.

[35] J. Lu and D. W. C. Ho, "Stabilization of complex dynamical networks with noise disturbance under performance constraint," Nonlinear Analysis: Real World Applications, vol. 12, no. 4, pp. 1974-1984, 2011.

[36] G.-P. Jiang, W. K.-S. Tang, and G. Chen, "A state-observer-based approach for synchronization in complex dynamical networks," IEEE Transactions on Circuits and Systems I: Regular Papers, vol. 53, no. 12, pp. 2739-2745, 2006.

[37] J. Wu and L. Jiao, "Observer-based synchronization in complex dynamical networks with nonsymmetric coupling," Physica A:
Statistical Mechanics and Its Applications, vol. 386, pp. 469-480, 2007.

[38] W. Ren and R. W. Beard, "Consensus seeking in multiagent systems under dynamically changing interaction topologies," IEEE Transactions on Automatic Control, vol. 50, no. 5, pp. 655661, 2005.

[39] G. Wen, Z. Duan, W. Yu, and G. Chen, "Consensus of multiagent systems with nonlinear dynamics and sampled-data information: a delayed-input approach," International Journal of Robust and Nonlinear Control, vol. 23, no. 6, pp. 602-619, 2013.

[40] R. A. Horn and C. R. Johnson, Matrix Analysis, Cambridge University Press, Cambridge, UK, 1985. 


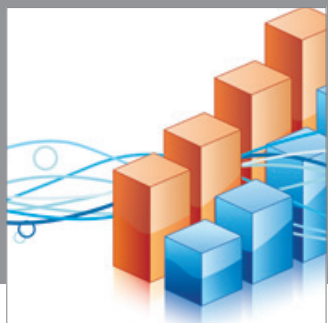

Advances in

Operations Research

mansans

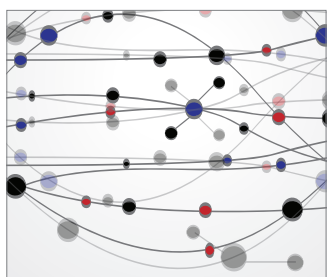

The Scientific World Journal
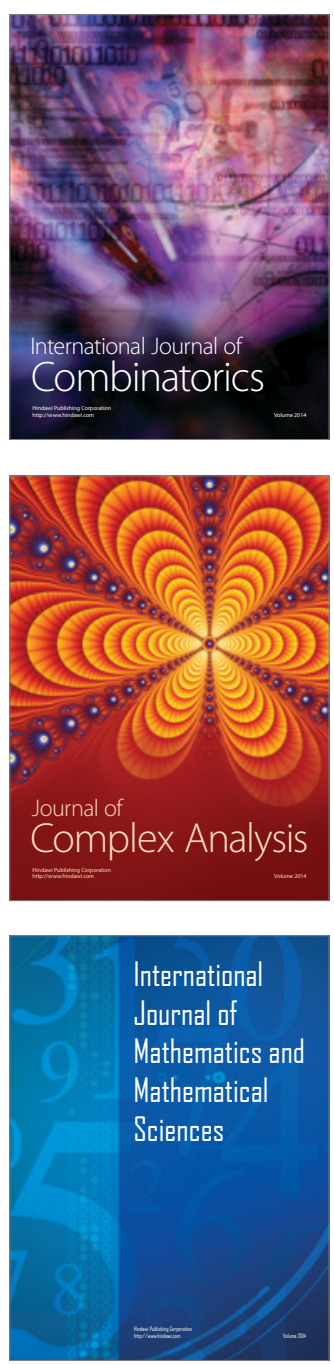
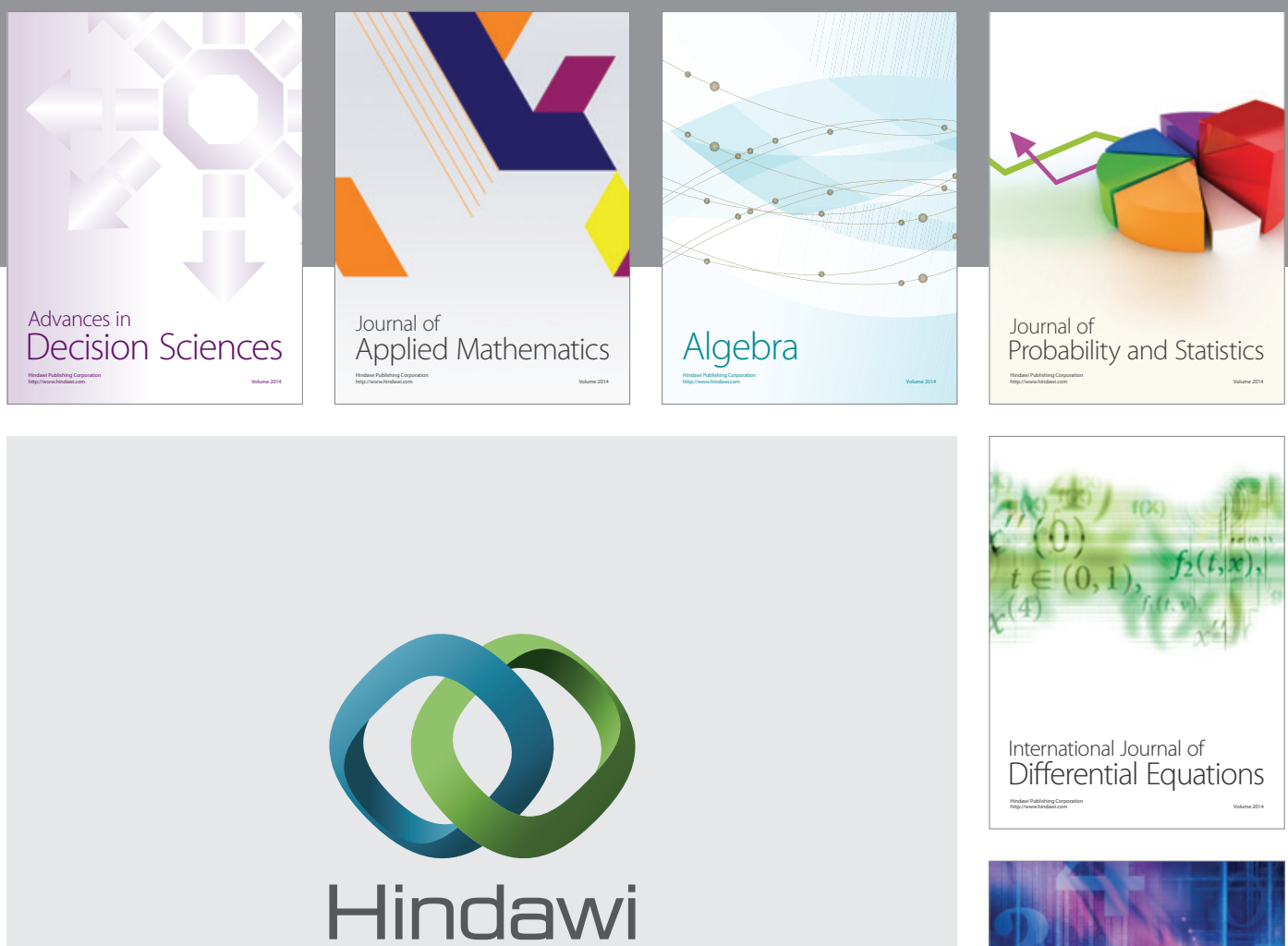

Submit your manuscripts at http://www.hindawi.com
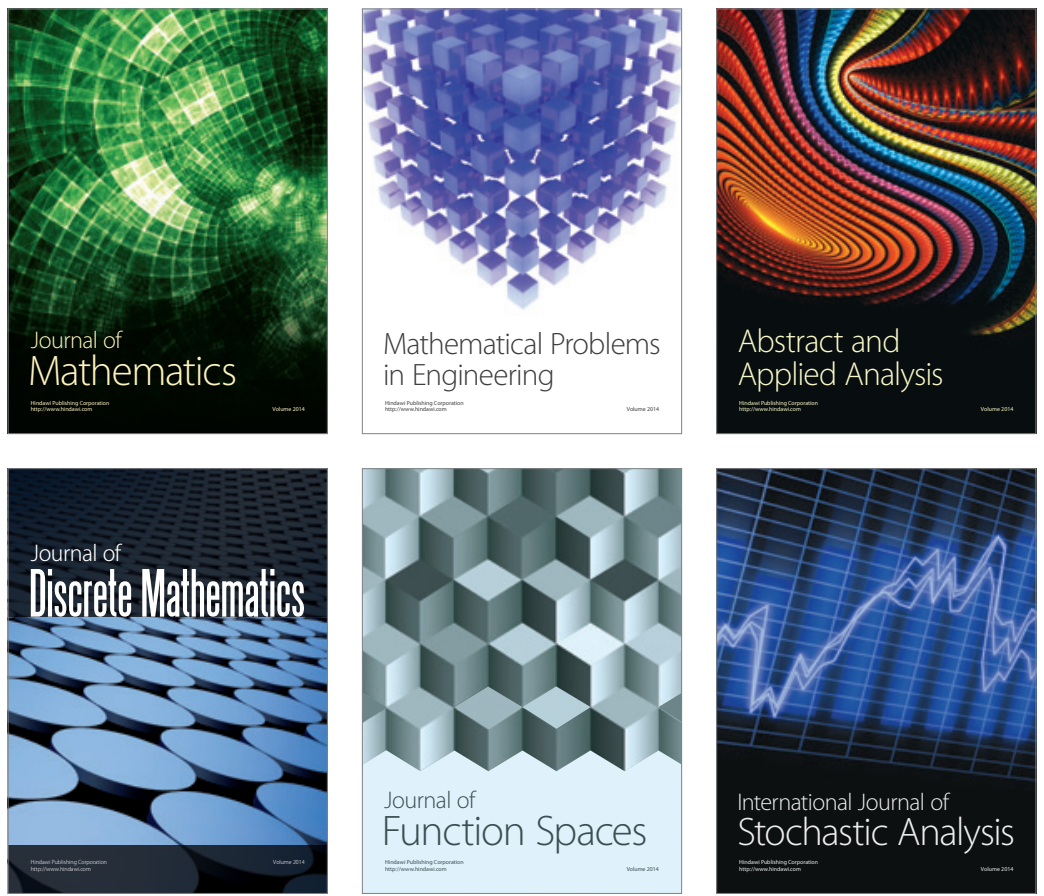

Journal of

Function Spaces

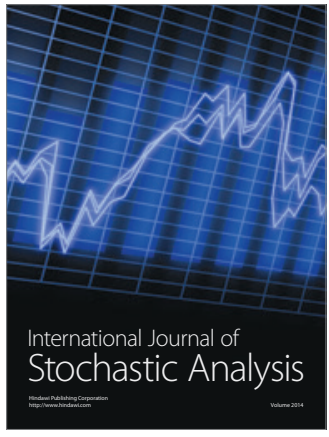

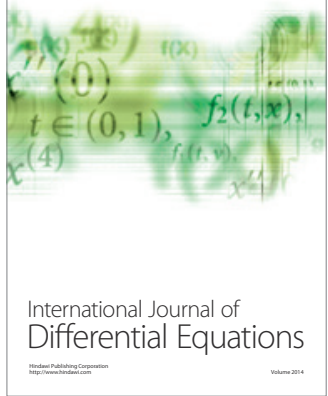
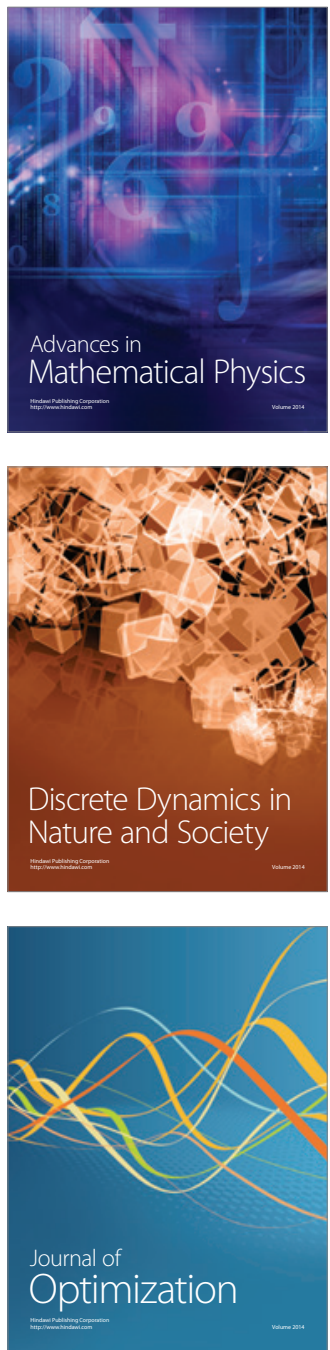